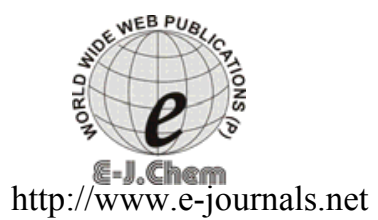

ISSN: 0973-4945; CODEN ECJHAO

E-Journal of Chemistry

2011, 8(S1), S137-S142

\title{
One-Pot Synthesis and Antimicrobial Activity of Novel $\alpha$-Aminophosphonates Using TMG
}

\author{
B. SIVA KUMAR*, Y. HARANADHA REDDY, \\ C. RADHA RANI and G. CHNADRA SEKHAR REDDY \\ * Department of Chemistry \\ Vel Tech Engineering College, Avadi, Chennai-600 062, India \\ Department of Chemistry \\ Sri Venakateswara University, Tirupati-517 502, India \\ bskumar_badur@yahoo.com
}

Received 2 March 2011; Accepted 28 April 2011

\begin{abstract}
Aminophosphonates (4a-j) were synthesized in one-pot simultaneous reaction of 4-bromo-3-methyl benzenamine (1), dimethylphosphite (3) and different aromatic aldehydes (2a-j) by Kabachnik-Fields reaction in the presence of tetramethylguanidine (TMG) $(10 \mathrm{~mole} \%)$ as catalyst in toluene at reflux temperature afforded $\mathbf{4 a}-\mathbf{j}$ in good yields. All these compounds were found to exhibit moderate to good antimicrobial activity.
\end{abstract}

Keywords: $\alpha$-Aminophosphonate, 4-Bromo3-methylbenzenamine, Tramethyl-guanidine, Antimicrobial activity

\section{Introduction}

Synthesis of $\alpha$-aminophosphonates exhibiting high bio-activity has recently attracted a lot of attention ${ }^{1-3}$. Their diverse applications include inhibition of synthase ${ }^{4}$, HIV protease ${ }^{5}$, renin $^{6}$, and PTPases ${ }^{7,8}$. Some of these derivatives are potential antibiotics ${ }^{9}$ and herbicides ${ }^{10}$. $\alpha$-Aminophosphonates are chief substrates in the synthesis of phosphonopeptides ${ }^{11}$. Due to their structural analogy with $\alpha$-amino acids, these types of organophosphorus compounds are widely used for the development of new inhibitors of enzymes, neuroactive compounds and plant growth regulators ${ }^{12,13}$. Among the number of synthetic approaches to $\alpha$-aminophosphonates, one of the most powerful methods is the Kabachnik-Fields reaction ${ }^{14,15}$. Previous results demonstrated that tetramethylguanidine (TMG) catalyzes the Michael addition of nitromethane to $\alpha, \beta$-unsaturated ketones ${ }^{16,17}$. TMG has been used only sporadically and has not yet received full recognition as a strong base in organic synthesis. Its catalytic activity in the Kabachnik-Fields reaction is explored in the present investigation. 


\section{Experimental}

IR spectra were recorded in $\mathrm{KBr}$ pellets on a Perkin-Elmer 683 spectrophotometer. ${ }^{1} \mathrm{H}$ NMR spectra were recorded at $300 \mathrm{MHz}$ in $\mathrm{CDCl}_{3}$ using TMS as internal standard reference. ${ }^{31} \mathrm{P}$ NMR $(121.4 \mathrm{MHz})$ was taken in $\mathrm{CDCl}_{3}$ using $85 \% \mathrm{H}_{3} \mathrm{PO}_{4}$ as external standard with broadband ${ }^{1} \mathrm{H}$ decoupling. ${ }^{13} \mathrm{C}$ NMR spectra measurements were performed at $75.4 \mathrm{MHz}$ using TMS as internal standard. ${ }^{1} \mathrm{H},{ }^{13} \mathrm{C},{ }^{31} \mathrm{P}$ NMR spectra were taken on Varian Gemini 300 $\mathrm{MHz}$ spectrometer and mass spectra were recorded on JEOL MSD- instrument.

\section{General procedure for the synthesis of $\alpha$ - aminophosphonates (4a-j)}

To a stirred solution of 4-bromo-3-methylbenzenamine (1) $(0.930 \mathrm{~g}, 0.005$ mole $)$ the aldehyde (2a-j) $(0.005$ mole $)$ in anhydrous toluene $(20 \mathrm{~mL})$ was added drop wise and then TMG (10 mole \%) was added and stirring continued at RT for $1 \mathrm{~h}$. Then dimethylphosphite (3) $(0.55 \mathrm{~g}, 0.005 \mathrm{~mole})$ in anhydrous toluene $(20 \mathrm{~mL})$ was added drop wise. Stirring was continued at RT for another $0.5 \mathrm{~h}$ and then the mixture was heated at gentle reflux for 5-6 h. The progress of the reaction was monitored by TLC analysis. After completion of the reaction the solvent was removed under reduced pressure. The residue was purified by column chromatography on silica gel (80-120 mesh) using hexane-ethyl acetate (8:2) as eluent.<smiles>[R]C(Nc1ccc(Br)c(C)c1)P(=O)(OC)OC</smiles>

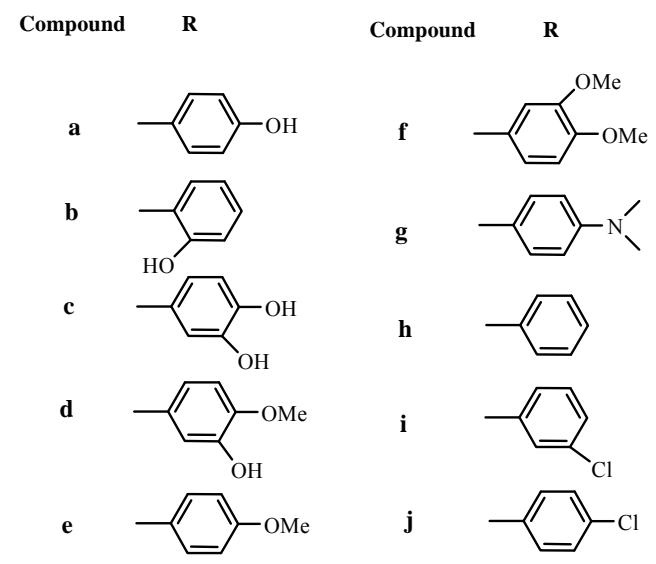

Scheme 1. Physical, analytical and spectral data for compounds $\mathbf{4 a - j}$

Dimethyl (4-bromo-3-methylphenylamino) (4-hydroxyphenyl) methylphosphonate (4a) Yield (68\%), mp 108-109 ${ }^{\circ} \mathrm{C}$; IR (KBr) cm ${ }^{-1} 3255(\mathrm{P}-\mathrm{N}-\mathrm{H}), 1233(\mathrm{P}=\mathrm{O}), 762$ (P-CH); ${ }^{1} \mathrm{H}-\mathrm{NMR}\left(\mathrm{DMSO}-d_{6}\right): \delta$ 6.12-7.09 $(\mathrm{m}, 7 \mathrm{H}, \mathrm{Ar}-\mathrm{H}), 4.98(\mathrm{~s}, 1 \mathrm{H}, \mathrm{NH}), 4.62-4.69(\mathrm{~m}, 1 \mathrm{H}$, $\mathrm{PCH}), 3.37-3.42\left(\mathrm{~d},{ }^{3} J_{\mathrm{P}-\mathrm{H}}=10.2 \mathrm{~Hz}, \mathrm{P}-\mathrm{OCH}_{3}\right), 3.52-3.64\left(\mathrm{~d},{ }^{3} \mathrm{~J}_{\mathrm{P}-\mathrm{H}}=10.9 \mathrm{~Hz}, \mathrm{P}-\mathrm{OCH}_{3}\right), 2.26$ (s, 3H, Ar-CH $\mathrm{CH}_{3}$ ); ${ }^{13} \mathrm{C}-\mathrm{NMR}\left(\mathrm{DMSO}-d_{6}\right), \delta 146.5$ (C-1), 112.7 (C-2), 132.5 (C-3), 112.4 (C-5), 139.1 
(C-5), 115.7 (C-6), 128.6 (C-1'), 136.5 (2C, C-2'\&6'), 115.5 (2C, C-3'\&5'), 156.9 (C-4'), $54.90(\mathrm{~d}$, $\mathrm{P}-\mathrm{CH}, J=7.6 \mathrm{~Hz}), 53.50\left(\mathrm{~d}, J=6.3 \mathrm{~Hz}, \mathrm{P}-\mathrm{OCH}_{3}\right) ;{ }^{31} \mathrm{P}-\mathrm{NMR}\left(\mathrm{DMSO}-d_{6}\right): \delta 26.28$; GCMS $\mathrm{m} / \mathrm{z}$ (\%): 401(M+2), 399(M+), 385.0 (11), 277.2 (100), 231.1 (15), 185.0 (36), 110.1 (4); Anal. Calcd. for $\mathrm{C}_{16} \mathrm{H}_{19} \mathrm{BrNO}_{4} \mathrm{P}: \mathrm{C}, 48.02 ; \mathrm{H}, 4.79 ; \mathrm{N}, 3.50$. Found: $\mathrm{C}, 47.91 ; \mathrm{H}, 4.73 ; \mathrm{N}, 3.47 \%$

Dimethyl (4-bromo-3-methylphenylamino) (2-hydroxyphenyl) methylphosphonate (4b)

Yield (73\%) mp 109-110 ${ }^{\circ} \mathrm{C}$; IR ( $\left.\mathrm{KBr}\right) \mathrm{cm}^{-1}$; 3375 (P-N-H), $1216(\mathrm{P}=\mathrm{O}), 756(\mathrm{P}-\mathrm{CH}) ;{ }^{1} \mathrm{H}-\mathrm{NMR}$ $\left(\mathrm{DMSO}-d_{6}\right) \delta$ 6.41-7.32 (m, 7H, Ar-H), 4.97 (s, 1H, NH), 4.68-4.80 (m, 1H,P-CH), 3.42-3.51 $\left(\mathrm{d},{ }^{3} \mathrm{~J}_{\mathrm{P}-\mathrm{H}}=10.1 \mathrm{~Hz}, \mathrm{P}-\mathrm{OCH}_{3}\right), 3.63-3.72\left(\mathrm{~d},{ }^{3} \mathrm{~J}_{\mathrm{P}-\mathrm{H}}=10.7 \mathrm{~Hz}, \mathrm{P}-\mathrm{OCH}_{3}\right) 2.31\left(\mathrm{~s}, 3 \mathrm{H}, \mathrm{Ar}_{-}-\mathrm{CH}_{3}\right)$; ${ }^{13} \mathrm{C}-\mathrm{NMR}$ (DMSO- $\left.d_{6}\right), \delta 145.9$ (C-1), 113.1 (C-2), 133.0 (C-3), 113.2 (C-4), 140.1 (C-5), 115.9 (C-6), 121.6 (C-1'), 154.5 (C-2'), 115.7 (C-3'), 128.9 (C-4') 121.7 (C-5'), 128.5 (C-6') 55.60 (d, $J=7.5 \mathrm{~Hz}, \mathrm{P}-\mathrm{CH}), 53.91\left(\mathrm{~d}, J=6.5 \mathrm{~Hz}, \mathrm{P}-\mathrm{OCH}_{3}\right) ;{ }^{31} \mathrm{P}-\mathrm{NMR}$ (DMSO- $\left.d_{6}\right): \delta 29.37$; Anal. Calcd. for $\mathrm{C}_{16} \mathrm{H}_{19} \mathrm{BrNO}_{4} \mathrm{P}: \mathrm{C}, 48.02 ; \mathrm{H}, 4.79 ; \mathrm{N}, 3.50$. Found: C,47.89 H,4.73 ; N,3.47\%

Dimethyl (4-bromo-3-methylphenylamino) (3,4-dihydroxyphenyl) methyl pho sphonate (4c)

Yield (71\%), mp 105-106 ${ }^{\circ} \mathrm{C}$; IR (KBr) cm${ }^{-1}$; $3345(\mathrm{P}-\mathrm{NH}), 1213(\mathrm{P}=\mathrm{O}), 775(\mathrm{P}-\mathrm{CH})$; ${ }^{1} \mathrm{H}-\mathrm{NMR}\left(\mathrm{DMSO}-d_{6}\right) \delta$ 6.56-7.51 (m, 6H, Ar-H), $4.87(\mathrm{~s}, 1 \mathrm{H}, \mathrm{NH}), 4.71-4.82(\mathrm{~m}, 1 \mathrm{H}, \mathrm{P}-$ $\mathrm{CH}), 3.38-3.47\left(\mathrm{~d},{ }^{3} \mathrm{~J}_{\mathrm{P}-\mathrm{H}}=10.1 \mathrm{~Hz}, \mathrm{P}-\mathrm{OCH}_{3}\right), 3.66-3.74\left(\mathrm{~d},{ }^{3} \mathrm{~J}_{\mathrm{P}-\mathrm{H}}=10.9 \mathrm{~Hz}, \mathrm{P}-\mathrm{OCH}_{3}\right), 2.35$ $\left(\mathrm{s}, 3 \mathrm{H}, \mathrm{Ar}-\mathrm{CH}_{3}\right) ;{ }^{13} \mathrm{C}-\mathrm{NMR}\left(\mathrm{DMSO}-d_{6}\right), \delta 146.9(\mathrm{C}-1), 114.9$ (C-2), 132.7 (C-3), $113.2(\mathrm{C}-4)$, 139.1 (C-5), 115.3 (C-6), 130.8 (C-1'), 114.3 (C-2'), 147.7 (C-3'), 145.9 (C-4') 117.5 (C-5'),

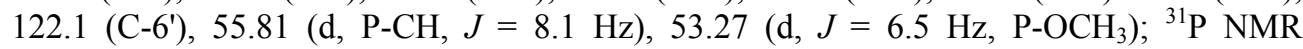
$\left(\right.$ DMSO- $\left.d_{6}\right) ; \delta$ 25.58. Anal. Calcd. for $\mathrm{C}_{16} \mathrm{H}_{19} \mathrm{BrNO}_{5} \mathrm{P}: \mathrm{C}, 44.80 ; \mathrm{H}, 4.26 ; \mathrm{N}, 3.48$. Found: C, $44.68 ; \mathrm{H}, 4.21 ; \mathrm{N}, 3.43 \%$.

Dimethyl (4-bromo-3-methylphenylamino) (3-hydroxy-4-methoxyphenyl) methyl phosphonate $(\mathbf{4 d})$

Yield (68\%), mp 97-98 ${ }^{\circ} \mathrm{C}$; IR (KBr) cm ${ }^{-1} ; 3390(\mathrm{P}-\mathrm{NH}), 1232(\mathrm{P}=\mathrm{O}), 742(\mathrm{P}-\mathrm{CH}) ; 1 \mathrm{H}$ NMR $\left(\mathrm{DMSO}-d_{6}\right) \delta$ 6.62-7.37 (m, 6H, Ar-H), $4.86(\mathrm{~s}, 1 \mathrm{H}, \mathrm{NH}), 4.66-4.78(\mathrm{~m}, 1 \mathrm{H}, \mathrm{PCH}), 3.81$ $\left(\mathrm{s}, 3 \mathrm{H}, \mathrm{Ar}-\mathrm{OCH}_{3}\right), 3.23-3.28\left(\mathrm{~d},{ }^{3} J_{\mathrm{P}-\mathrm{H}}=10.0 \mathrm{~Hz}, \mathrm{P}-\mathrm{OCH}_{3}\right), 3.57-3.65\left(\mathrm{~d},{ }^{3} J_{\mathrm{P}-\mathrm{H}}=11.1 \mathrm{~Hz}\right.$, $\mathrm{P}^{\left.-\mathrm{OCH}_{3}\right)} 2.28\left(\mathrm{~s}, 3 \mathrm{H}, \mathrm{Ar}-\mathrm{CH}_{3}\right) ;{ }^{13} \mathrm{C}$ NMR (DMSO-d $\left.d_{6}\right) \delta 122.7(\mathrm{C}-2), 112.5(\mathrm{C}-3), 121.8$ (C-4), 120.6 (C-5), 119.6 (C-6), 111.5 (C-7), 131.2 (C-8), 179 (C-9), 151.5 (C-3'), 113.5 (C-4'), $160.9\left(\mathrm{C}-5^{\prime}\right), 55.83(\mathrm{~d}, J=7.9 \mathrm{~Hz}, \mathrm{P}-\mathrm{CH}), 54.0\left(\mathrm{~d}, J=6.4 \mathrm{~Hz}, \mathrm{P}-\mathrm{OCH}_{3}\right), 15.8$ $\left(\mathrm{Ar}-\mathrm{CH}_{3}\right) ;{ }^{31} \mathrm{P}$ NMR (DMSO-d $) ; \delta 31.29$; Anal. Calcd. for $\mathrm{C}_{17} \mathrm{H}_{21} \mathrm{BrNO}_{5} \mathrm{P}: \mathrm{C}, 47.46 ; \mathrm{H}$, 4.92; N, 3.26. Found: C, 47.34; H, 4.85; N, 3.19\%.

Dimethyl (4-bromo-3-methylphenylamino) (4-methoxyphenyl) methyl phosphonate (4e)

Yield (74\%), mp 110-111 ${ }^{\circ} \mathrm{C}$; IR (KBr) cm ${ }^{-1}$; $3225(\mathrm{P}-\mathrm{NH}), 1228(\mathrm{P}=\mathrm{O}), 750(\mathrm{P}-\mathrm{CH})$; ${ }^{1} \mathrm{H}-\mathrm{NMR}\left(\mathrm{DMSO}-d_{6}\right)$ 86.72-7.54 (m, 7H, Ar-H), $5.08(\mathrm{~s}, 1 \mathrm{H}, \mathrm{NH}), 4.75-4.93(\mathrm{~m}, 1 \mathrm{H}$, $\mathrm{PCH}), 3.87\left(\mathrm{~s}, 3 \mathrm{H}, \mathrm{Ar}-\mathrm{OCH}_{3}\right), 3.22-3.29\left(\mathrm{~d},{ }^{3} J_{\mathrm{P}-\mathrm{H}}=10.0 \mathrm{~Hz}, \mathrm{P}-\mathrm{OCH} 3\right), 3.61-3.68\left(\mathrm{~d},{ }^{3} J_{\mathrm{P}-}\right.$ $\left.{ }_{\mathrm{H}}=11.2 \mathrm{~Hz}, \mathrm{P}-\mathrm{OCH}_{3}\right), 2.15\left(\mathrm{~s}, 3 \mathrm{H}, \mathrm{Ar}-\mathrm{CH}_{3}\right) ;{ }^{31} \mathrm{P}$ NMR (DMSO- $\left.d_{6}\right) ; \delta 28.62$. Anal. Calcd. for $\mathrm{C}_{17} \mathrm{H}_{21} \mathrm{BrNO}_{4} \mathrm{P}: \mathrm{C}, 49.29 ; \mathrm{H}, 5.11 ; \mathrm{N}, 3.38$. Found: C, 49.17; H, 5.07; N, 3.32\%.

Dimethyl (4-bromo-3-methylphenylamino) (3,4-dimethoxyphenyl) methyl phosphonate (4f)

Yield (69\%), mp 123-124 ${ }^{\circ} \mathrm{C}$; IR (KBr) cm-1; 3385 (P-NH), $1235(\mathrm{P}=\mathrm{O}), 762(\mathrm{P}-\mathrm{CH})$; ${ }^{1} \mathrm{H}-\mathrm{NMR}\left(\mathrm{DMSO}-d_{6}\right)$ 86.60-7.64 (m, 6H, Ar-H), 4.97 (s, 1H, NH), 4.85-4.89 (m, 1H, P$\mathrm{CH}), 3.87-3.98\left(\mathrm{~m} \mathrm{6H}, \mathrm{Ar}-\mathrm{OCH}_{3}\right), 3.29-3.37\left(\mathrm{~d},{ }^{3} J_{\mathrm{P}-\mathrm{H}}=10.0 \mathrm{~Hz}, \mathrm{P}-\mathrm{OCH}_{3}\right), 3.59-3.68\left(\mathrm{~d},{ }^{3} J_{\mathrm{P}-}\right.$ $\left.{ }_{\mathrm{H}}=11.1 \mathrm{~Hz}, \mathrm{P}-\mathrm{OCH}_{3}\right) ; 2.37\left(\mathrm{~s}, 3 \mathrm{H}, \mathrm{Ar}-\mathrm{CH}_{3}\right) .{ }^{31} \mathrm{P} \mathrm{NMR}\left(\mathrm{DMSO}-d_{6}\right) ; \delta 26.76$. Anal. Calcd. for $\mathrm{C}_{18} \mathrm{H}_{23} \mathrm{BrNO}_{5} \mathrm{P}$ : C, 48.66; H, 5.22; N, 3.15. Found: C, 48.55; H,5.16; N, 3.08\%. 
Dimethyl(4-bromo-3-methylphenylamino)(4-N, N-dimethylphenyl) methyl phosphonate (4g) Yield (60\%), mp 145-146 ${ }^{\circ} \mathrm{C}$; IR (KBr) cm${ }^{-1}$; $3385(\mathrm{P}-\mathrm{NH}), 1238(\mathrm{P}=\mathrm{O}), 758(\mathrm{P}-\mathrm{CH})$; ${ }^{1} \mathrm{H}-\mathrm{NMR}\left(\mathrm{DMSO}-d_{6}\right) \delta$ 6.72-7.58 (m, 7H, Ar-H), $5.14(\mathrm{~s}, 1 \mathrm{H}, \mathrm{NH}), 4.86-4.90(\mathrm{~m}, 1 \mathrm{H}, \mathrm{P}-\mathrm{CH})$; 3.31-3.39 (d, $\left.{ }^{3} J_{\mathrm{P}-\mathrm{H}}=10.1 \mathrm{~Hz}, \mathrm{P}-\mathrm{OCH}_{3}\right) ; 3.55-3.65\left(\mathrm{~d},{ }^{3} J_{\mathrm{P}-\mathrm{H}}=11.3 \mathrm{~Hz}, \mathrm{P}-\mathrm{OCH}_{3}\right), 2.84$ $\left(\mathrm{S}, 6 \mathrm{H}, \mathrm{N}\left(\mathrm{CH}_{3}\right)_{2}\right), 2.28\left(\mathrm{~s}, 3 \mathrm{H}, \mathrm{Ar}-\mathrm{CH}_{3}\right) ;{ }^{31} \mathrm{P} \mathrm{NMR}$ (DMSO-d $\left.)_{6}\right) ; \delta: 31.30$. Anal. Calcd. for $\mathrm{C}_{18} \mathrm{H}_{24} \mathrm{BrN}_{2} \mathrm{O}_{3} \mathrm{P}:$ C, 50.60; H, 5.66; N, 6.56. Found: C, 50.48; H, 5.57; N, 6.51\%.

Dimethyl (4-bromo-3-methylphenylamino) (phenyl) methylphosphonate (4h) Yield $(69 \%), \mathrm{mp} 143-144^{\circ} \mathrm{C}$; IR $(\mathrm{KBr}) \mathrm{cm}^{-1} ; 3387(\mathrm{P}-\mathrm{NH}), 1232(\mathrm{P}=\mathrm{O}), 755(\mathrm{P}-\mathrm{CH})$; ${ }^{1} \mathrm{H}-\mathrm{NMR}\left(\mathrm{DMSO}-d_{6}\right) \delta$ 6.52-7.66 (m, 8H, Ar-H), $5.04(\mathrm{~s}, 1 \mathrm{H}, \mathrm{NH}), 4.70-4.85(\mathrm{~m}, 1 \mathrm{H}, \mathrm{P}-\mathrm{CH})$, 3.29-3.41 (d, $\left.{ }^{3} J_{\mathrm{P}-\mathrm{H}}=10.0 \mathrm{~Hz}, \mathrm{P}-\mathrm{OCH}_{3}\right) ; 3.54-3.63\left(\mathrm{~d},{ }^{3} J_{\mathrm{P}-\mathrm{H}}=11.2 \mathrm{~Hz}, \mathrm{P}-\mathrm{OCH}_{3}\right) 2.26(\mathrm{~s}, 3 \mathrm{H}$, Ar- $\left.\mathrm{CH}_{3}\right) ;{ }^{31} \mathrm{P}$ NMR (DMSO- $\left.d_{6}\right) \delta 30.30$. GCMS $m / z(\%): 384(\mathrm{M}+), 385.0(8), 369.4(16)$, 262.3 (100), 215.6 (18), 172.5 (9), 110.2 (5): Anal.Calcd. for $\mathrm{C}_{16} \mathrm{H}_{19} \mathrm{BrNO}_{3} \mathrm{P}: \mathrm{C}, 50.02 ; \mathrm{H}$, 4.98 ; N, 3.65. Found: C, 49.91; H, 4.91; N, 3.61\%.

Dimethyl (4-bromo-3-methylphenylamino) (3-chlorophenyl) methylphosphonate (4i) Yield (73\%), mp 161-162 ${ }^{\circ}$; IR (KBr) $\mathrm{cm}^{-1}$; $3350(\mathrm{P}-\mathrm{NH}), 1218(\mathrm{P}=\mathrm{O}), 780(\mathrm{P}-\mathrm{CH})$; ${ }^{1} \mathrm{H}$ NMR(DMSO- $\left.d_{6}\right) \delta: 6.53-7.76(\mathrm{~m}, 7 \mathrm{H}, \mathrm{Ar}-\mathrm{H}), 5.03(\mathrm{~s}, 1 \mathrm{H}, \mathrm{NH}), 4.76-4.80(\mathrm{~m}, 1 \mathrm{H}$, P-CH), 3.36-3.44 (d, $\left.{ }^{3} J_{\mathrm{P}-\mathrm{H}}=10.1 \mathrm{~Hz}, \mathrm{P}-\mathrm{OCH}_{3}\right) ; 3.61-3.69\left(\mathrm{~d},{ }^{3} J_{\mathrm{P}-\mathrm{H}}=11.1 \mathrm{~Hz}, \mathrm{P}-\mathrm{OCH}_{3}\right), 2.21$ (s, $\left.3 \mathrm{H}, \mathrm{Ar}-\mathrm{CH}_{3}\right) ;{ }^{31} \mathrm{P}$ NMR(DMSO-d $\left.d_{6}\right) \delta 20.18$. Anal. Calcd. for $\mathrm{C}_{16} \mathrm{H}_{18} \mathrm{BrClNO}_{3} \mathrm{P}: \mathrm{C}, 45.90$; $\mathrm{H}, 4.33$; N, 3.35. Found: C, 45.81; H, 4.27; N, 3.29\%.

Dimethyl (4-bromo-3-methylphenylamino) (4-chlorophenyl) methylphosphonate (4j) Yield (74\%), mp 157-158 ${ }^{\circ} \mathrm{C}$; IR (KBr) cm $\mathrm{cm}^{-1} 3372(\mathrm{P}-\mathrm{NH}), 1211(\mathrm{P}=\mathrm{O}), 776(\mathrm{P}-\mathrm{CH})$. ${ }^{1} \mathrm{H}-\mathrm{NMR}\left(\mathrm{DMSO}-d_{6}\right) \delta$ 6.75-7.54(m, 7H, Ar-H), $5.12(\mathrm{~s}, 1 \mathrm{H}, \mathrm{NH}), 4.79-4.84(\mathrm{~m}, 1 \mathrm{H}$, P-CH), 3.42-3.49 (d, $\left.{ }^{3} J_{\mathrm{P}-\mathrm{H}}=9.8 \mathrm{~Hz}, \mathrm{P}-\mathrm{OCH}_{3}\right), 3.71-3.78\left(\mathrm{~d},{ }^{3} J_{\mathrm{P}-\mathrm{H}}=10.5 \mathrm{~Hz}, \mathrm{P}-\mathrm{OCH}_{3}\right), 2.17$ (m, 3H, Ar- $\mathrm{CH}_{3}$ ); ${ }^{31} \mathrm{P}$ NMR (DMSO- $\left.d_{6}\right)$ 831.80. Anal. Calcd. for $\mathrm{C}_{16} \mathrm{H}_{18} \mathrm{BrClNO}_{3} \mathrm{P}: \mathrm{C}$, 45.90; H, 4.33; N, 3.35. Found: C, 45.78; H, 4.25; N, 3.28\%.

\section{Results and Discussion}

4-Bromo 3-methyl benzenamine (1) was treated with different aldehydes (2a-j) and dimethyl phosphite (3) in the presence of 10 mole \% of tetramethylguanidine (TMG) in dry toluene at RT. The mixture was stirred at RT for $1 \mathrm{~h}$ and at $70-80{ }^{\circ} \mathrm{C}$ for another $5 \mathrm{~h}$. The progress of the reaction was monitored by thin layer chromatography. The reaction proceeded smoothly, and completed in 5-6 $\mathrm{h}$ to afford the corresponding $\alpha$ aminophosphonates in high yield (60-74\%). This showed that TMG acts as an effective catalyst in this reaction. An important feature is that the TMG can be easily recovered from the reaction mixture after completion of the reaction and can be reused. The chemical structures of all the new compounds were confirmed by elemental analysis, IR, ${ }^{1} \mathrm{H},{ }^{13} \mathrm{C}$ and ${ }^{31} \mathrm{P}$ NMR spectra. Compounds $\mathbf{4 a - j}$ exhibited characteristic IR stretching frequencies in the regions $3225-3390,1211-1238,742-780 \mathrm{~cm}^{-1}$ for $\mathrm{N}-\mathrm{H}, \mathrm{P}=\mathrm{O}$ and $\mathrm{P}-$ $\mathrm{C}$ (aliphatic) respectively ${ }^{18}$. The aromatic protons of the benzene rings of the $\alpha$-aminophosphonates (4a-j) showed a complex multiplet at $\delta$ 6.12-7.76. The P-C-H proton signal appeared as a multiplet ${ }^{19}$ at $\delta 4.62-4.93$ due to its coupling with both phosphorus and the $\mathrm{N}-\mathrm{H}$ proton. The exocyclic N-H proton signal appeared at $\delta 4.87-5.14$ as a singlet. The methoxy group protons of the dimethylphosphite moiety resonated as two distinct doublets in the range of $\delta 3.22-3.49\left(\mathrm{~d},{ }^{3} J_{\mathrm{P}-\mathrm{H}}=9.8-10.1 \mathrm{~Hz}\right)$ and $\delta 3.54-3.79\left(\mathrm{~d},{ }^{3} J_{\mathrm{P}-\mathrm{H}}=\right.$ $10.50-11.30)^{19,20}$ indicating their non-equivalence. The carbon chemical shifts for P-CH-, $\mathrm{P}-\mathrm{O}-\mathrm{CH}_{3}$ 
in the title compounds were observed at $\delta 54.90-55.83,53.27-54.00$ respectively $^{18,21,22}$. The ${ }^{31} \mathrm{P}$ NMR signals ${ }^{23}$ appeared in the region $\delta 20.18-31.80$ for these compounds.

\section{Antimicrobial activity}

The antibacterial activity of (4a-j) was assayed ${ }^{24}$ against the growth of staphylococcus aureus (gram +ve) and Escherichia coli (gram -ve) at three concentrations (100, 50, 25 ppm) (Table 1). The majority of the compounds exhibited moderate to good activity against both the bacteria. The same (4a-j) were screened for their antifungal activity ${ }^{25}$ (Table 2) against Aspergillus niger and Helminthosporium oryzae species along with the standard fungicide Griseofulvin at three different concentrations $(100,50,25 \mathrm{ppm})$. It is gratifying to observe that the majority of the compounds $\mathbf{4 a - j}$ exhibited moderate to good antifungal activity when compared with the Griseofulvin reference.

Table 1. Antibacterial activity of $\alpha$-aminophosphonates (4a-j)

\begin{tabular}{ccccccc}
\hline & \multicolumn{6}{c}{ Zone of inhibition, \% } \\
\cline { 2 - 7 } Compound & \multicolumn{5}{c}{ Escherichia coli } & \multicolumn{5}{c}{ Staphylococcus aureus } \\
\cline { 2 - 7 } & 100 & 50 & 25 & 100 & 50 & 25 \\
\hline $\mathbf{4 a}$ & 08 & 05 & 02 & 09 & 05 & 01 \\
$\mathbf{4 b}$ & 07 & 05 & 03 & 09 & 04 & 02 \\
$\mathbf{4 c}$ & 09 & 04 & - & 08 & 04 & - \\
$\mathbf{4 d}$ & 05 & 03 & 01 & 07 & 04 & - \\
$\mathbf{4 e}$ & 09 & 04 & - & 06 & 05 & - \\
$\mathbf{4 f}$ & 08 & 05 & 03 & 10 & 06 & 02 \\
$\mathbf{4 g}$ & 08 & 04 & - & 09 & 04 & 01 \\
$\mathbf{4 h}$ & 08 & 05 & - & 08 & 05 & 02 \\
$\mathbf{4 i}$ & 08 & 05 & 01 & 06 & 03 & - \\
$\mathbf{4 j}$ & 05 & 02 & - & 07 & 03 & - \\
Pencillin & 12 & 07 & - & 11 & 08 & - \\
\hline
\end{tabular}

Table 2. Antifungal activity of $\alpha$-aminophosphonates (4a-j)

\begin{tabular}{ccccccc}
\hline & \multicolumn{6}{c}{ Zone of inhibition, \% } \\
\cline { 2 - 7 } Compound & \multicolumn{7}{c}{ Aspergillus Niger } & \multicolumn{4}{c}{ Helmenthosphorium oryzae } \\
\cline { 2 - 7 } & 100 & 50 & 25 & 100 & 50 & 25 \\
\hline $\mathbf{4 a}$ & 07 & 05 & 03 & 10 & 06 & 03 \\
$\mathbf{4 b}$ & 07 & 05 & 03 & 08 & 05 & 02 \\
$\mathbf{4} \mathbf{c}$ & 09 & 06 & 04 & 09 & 05 & 03 \\
$\mathbf{4 d}$ & 05 & 03 & 01 & 07 & 04 & - \\
$\mathbf{4 e}$ & 09 & 04 & - & 06 & 05 & - \\
$\mathbf{4 f}$ & 08 & 05 & 03 & 10 & 06 & 02 \\
$\mathbf{4 g}$ & 08 & 04 & - & 09 & 04 & 01 \\
$\mathbf{4 h}$ & 09 & 04 & - & 08 & 04 & - \\
$\mathbf{4 i}$ & 08 & 05 & 02 & 09 & 05 & 01 \\
$\mathbf{4 j}$ & 07 & 03 & - & 08 & 04 & 01 \\
Griseo & 11 & 08 & - & 13 & 08 & - \\
fulvin & 11 & & & & & \\
\hline
\end{tabular}




\section{Acknowledgment}

The authors express their thanks to Prof C. Suresh Reddy, S.V. University, Tirupati for his suggestions and Dr. C. Chellaram Department of Biomedical Engineering, VelTech MultiTech Dr.R.R.\& Dr.S.R Engineering college for his help in biological studies. We extend our thanks to Director IISc Bangalore for providing spectral data.

\section{References}

1. Kaboudin B, Nazari R, Tetrahedron Lett., 2001, 42, 8211.

2. Matveeva E D, Podrugina T A, Tishkovskaya E V, Tomilova L G and Zefirov N S, Synlett., 2003, 2321-2324.

3. Pavlov V. Ya, Kabachinik M M, Zobnina E V, Ponomarev G V and Beletskaya I P, Synlett., 2003, 2193.

4. Sikorski J A, Miller M J, Braccolino D S, Cleary D G, Corey S D, Ream J E, Schnur D, Shah A and Walker M C, Phosphorus Sulfur Silicon., 1993, 76, 375.

5. Stowasser B, Budt K H, Jain-Qi L, Peyman A and Ruppert D, Tetrahedron Lett., 1992, 33, 6625.

6. Patel D V, Reilly-Gauvin K and Ryono D E, Tetrahedron Lett., 1990, 31, 5587.

7. (a) Bruke T R, Jr Brachi J J, Jr George C, Wolf G, Shoelson S E and Yan X, J Med Chem., 1995, 38, 1386-1396; (b) Bruke T R, Jr Kole H K and Roller P P, Biochem Biophys Res Commun., 1994, 204, 129-134.

8. Peyman A, Budt K H, Paning J S, Stowasser B and Ruppert D, Tetrahedron Lett., 1992, 33, 4549-4552.

9. Atherton F R, Hassall C H and Lambert R W, J Med Chem., 1986, 29, 29-40.

10. Kafarski P, Lejack B and Mastalerz P, Beitr Wirk Forsh., 1985, H25; Chem Abstr., 1985, 103, 174532.

11. (a) Maier L and Lea P J, Phosphorus Sulfur Silicon 1983, 17, 1; (b) Giannousis P P, Bartlett P A, J Med Chem., 1987, 30, 1603-1609; (c) Gancarz R and Wieczorek J S, Synthesis, 1977, 625; (d) Baylis E K, Campbell C D and Dingwall J G, J Chem Soc., PerkinTrans 1, 1984, 2845-2853.

12. Fields S C, Tetrahedron, 1999, 55, 12237.

13. Kleszczynska H and Sarapuk J, Cell Mol Biol Lett., 2001, 6(1), 83-91.

14. (a) Kabachinic M J and Medved T, Izv Akad Nauk SSSR, 1953, 1126; (b) Kabachinic M J and Medved T, Izv Akad Nauk SSSR, 1954, 1024.

15. Fields E K, J Am Chem Soc., 1952, 74, 1528.

16. Pollini G P, Barco A and Degiuli G, Synthesis, 1924, 44.

17. Hewson A. T and Macpherson D T, Tetrahedron Lett., 1983, 24, 647.

18. Silverstein R M and Webster F X, Spectrometric Identification of Organic Compounds, $6^{\text {th }}$ Edn., Wiley: New York, 1998.

19. Tongcharoensirikul P, Suarez A I, Voelker T and Thomson C M, J Org Chem., 2004, 69, 2322.

20. Heydari A, Zarei M, Alijaninzadeh R and Tavakol H, Tetrahedron Lett., 2001, 42, 3629.

21. Qian C and Huang, T, J Org Chem., 1998, 63, 4125.

22. Semenzin D, Moghadam G E, Albiouy D, Diallo O and Koenig M, J Org Chem., 1997, 62, 2414.

23. Quin L D and Verkade J G, Phosphorus-31 NMR Spectral Properties in Compound Characterization and Structural Analysis; VCH: New York, 1994

24. Vincent J C and Vincent H W, Proc Soc Expt Biol Med., 1944, 55, 162.

25. Benson H J, Microbiological Applications, $5^{\text {th }}$ Edn., Brown W C, Publications: Boston, 1990. 


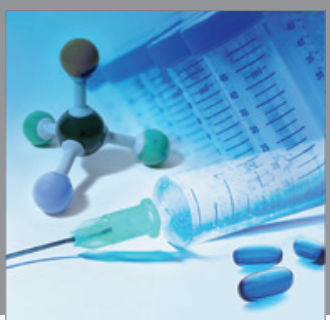

International Journal of

Medicinal Chemistry

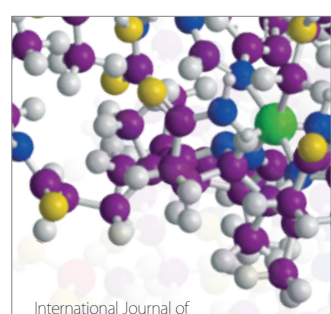

Carbohydrate Chemistry

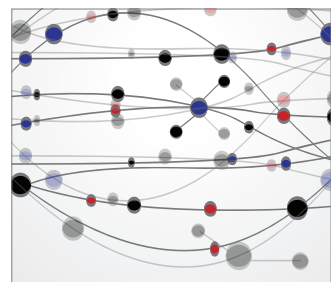

The Scientific World Journal
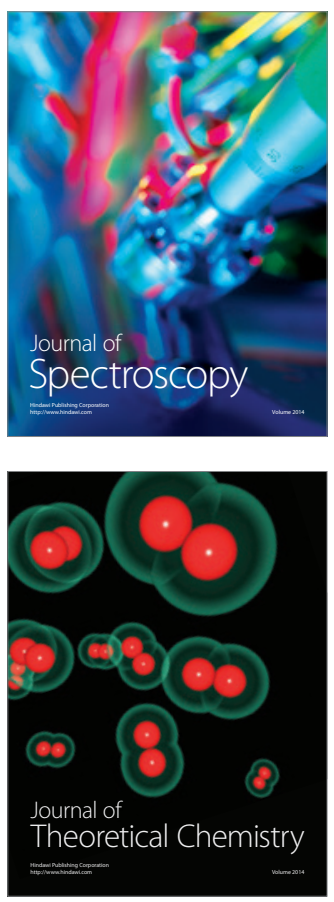
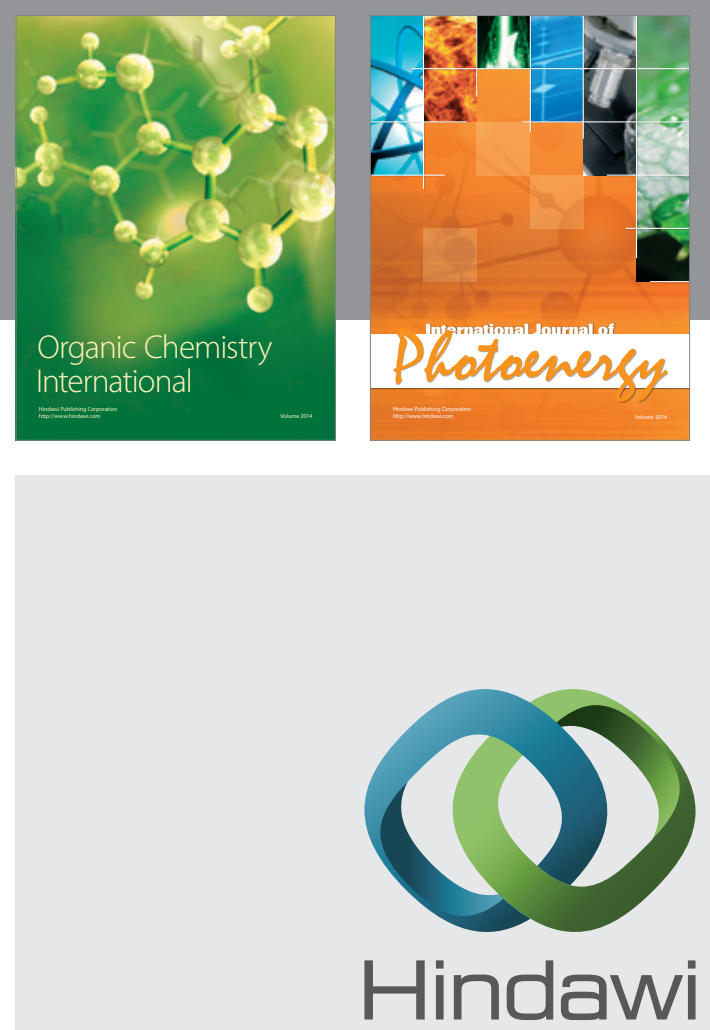

Submit your manuscripts at

http://www.hindawi.com
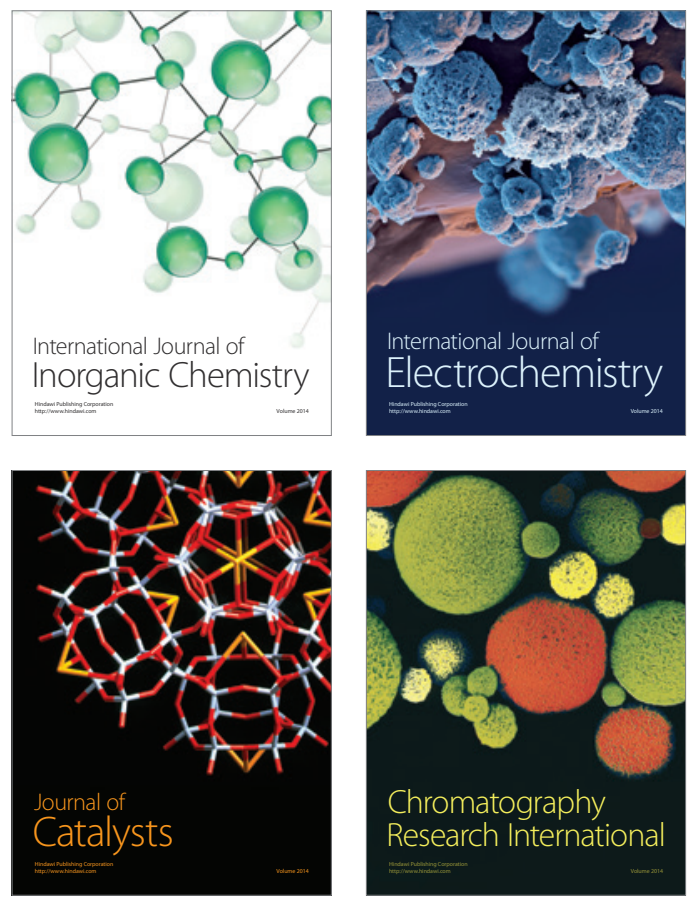
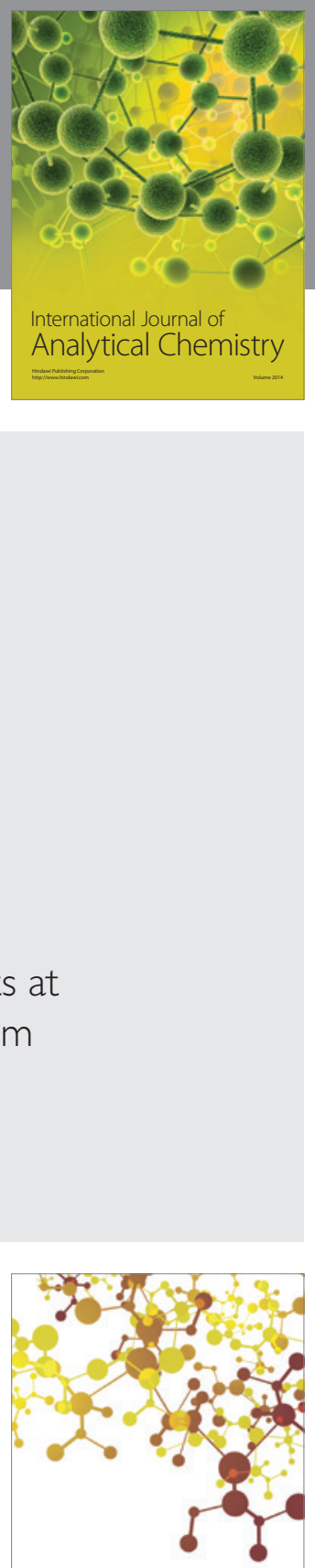

Journal of

Applied Chemistry
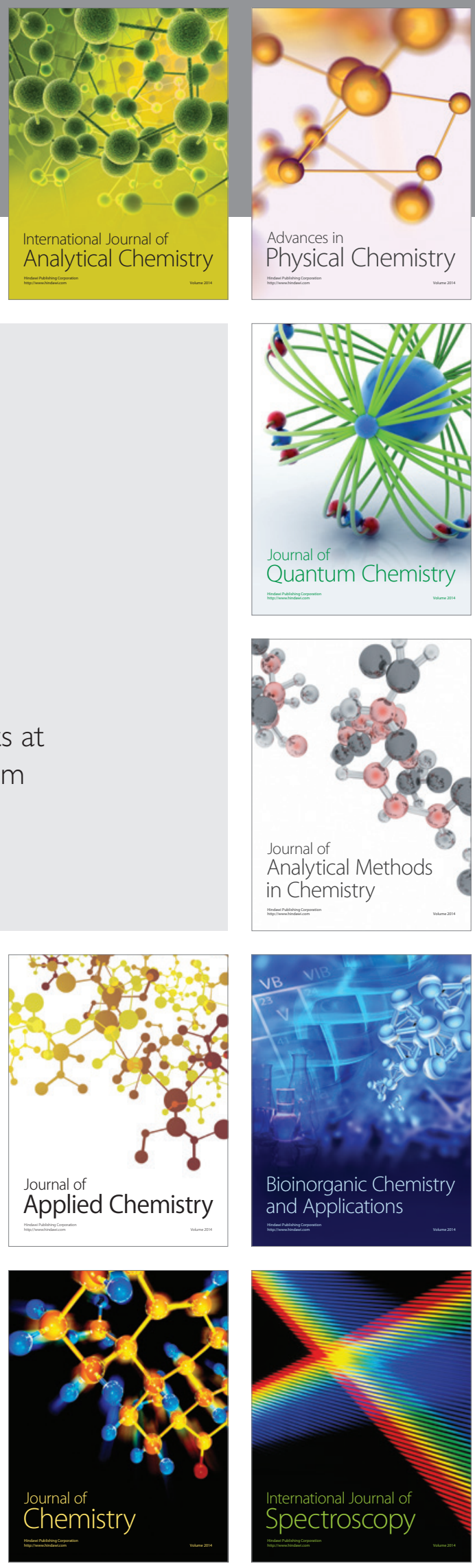